\title{
Astronomy Student Research in the International Baccalaureate
}

\author{
K. Ross Cutts ${ }^{1,2 *}$
}

\begin{abstract} are discussed.

${ }^{1}$ St. Paul's Grammar School, 52 Taylor Rd, Cranebrook NSW 2749 Australia

${ }^{2}$ Edith Cowan Institute for Educational Research, Joondalup, WA, 6027

*Corresponding author: krcutts@gmail.com
\end{abstract}

Our Solar Siblings has a rich history of promoting inquiry-based learning using robotic telescopes across a broad range of students around Australia. The author has delivered the Our Solar Siblings*(OSS) program to a range of students from Year 7-10 (12-16 years old) in Gifted and Talented Science classes. As part of the delivery, students have been encouraged to pursue individual astronomy research to enhance their understanding and skills of Physics concepts. Over the past 3 years, the Our Solar Siblings curriculum has also been delivered to senior physics students at St. Paul's Grammar School in New South Wales, Australia as part of their International Baccalaureate (IB) Diploma Programme (DP) Physics course (16-19 years old). The IB is delivered around the world as an alternative to traditional courses studied by students in their countries and aims to develop inquiring, knowledgeable and caring young people. Since Astrophysics is an Option in the IB DP Physics, OSS has been used as a platform to enthuse and encourage student participation. The match between student research and the IB curriculum is discussed in detail. Students have extended their involvement by pursuing their own areas of astronomy research with the assistance of an OSS mentor as their academic supervisor. The areas of research, benefits and challenges

\section{Introduction}

Astronomy research conducted by high school students has a long and rich history. Astronomy is one of the oldest scientific fields, yet it continues to capture the imagination of the young and old alike (Bailey and Lombardi, 2015). Although astronomy topics have been included in K-12 education research for decades, astronomy education research as a field of discipline-based education research is relatively new (Bailey, 2011). Similarly, the use of remote and robotic telescopes in education has had a relatively short life so far (Gomez and Fitzgerald, 2017). In recent years, calls for the adoption of inquiry-based pedagogies in the science classroom have formed a part of the recommendations for large-scale high school science reforms, despite their lack of implementation in the typical classroom (Danaia et al., 2013). This has not been without its difficulties with many teachers finding barriers to teaching inquiry-based science in the classroom (Fitzgerald et al., 2017). The International Baccalaureate (IB) Diploma Programme (DP) Physics course is founded on an inquiry-based pedagogy, and was seen as an ideal platform to trial student research in astronomy.

The author was originally involved in the Space to Grow project (Danaia et al., 2012), which was a three-year funded Australian Research Council (ARC) grant run through Macquarie University and Charles Sturt University with support from the Las Cumbres Observatory Global Telescope (LCOGT) 
network and three educational jurisdictions in New South Wales, Australia. The focus of the project was to get high school teachers to utilise the two 2-metre Faulkes telescopes in their classrooms to undertake authentic science. The Our Solar Siblings (OSS) initiative (Fitzgerald et al., 2018) was an easy transition to make from this earlier project.

OSS is a project based in Australia focused at bringing professional-grade telescope access into the high-school classroom to model authentic research. Interested students could then undertake their own authentic research-grade independent research projects (along the lines of Fitzgerald et al., 2014), usually on RRLyrae stars or Open Clusters, mentored by project staff and professional astronomers. It was this model that was implemented by the author and his students in this paper.

In a later paper on OSS, (Fitzgerald et al., 2016) highlighted the benefits of teacher training. Of particular note was the fact that the materials were ready to use in the classroom. After each training day, the teachers were capable of taking the material directly into their classrooms, as some of them did to use with their students. As the author had been trained in the Space to Grow project, and then OSS, it was a natural progression to trial OSS in the IB. With the support and encouragement of OSS, the author then embarked on a three year journey to deliver OSS to his senior IB Physics students, and then to encourage many of them to do further astronomy research for their compulsory IB work.

It is important to note, that to-date, there have never been any papers specifically addressing the suitability of the IB for astronomy education. The author was breaking new ground, and therefore the results below may hopefully encourage other IB Physics teachers to embark upon integrating astronomy research in their teaching programs.

\section{Astronomy and Student Enrichment}

The decision to deliver astronomy to science students was born out of a desire to attract more students to senior science courses, particularly Physics, and to extend the knowledge and skills of the students. Initially Space to Grow, then Our Solar Siblings, were used as the curriculum platforms. The author participated in a number of teacher-training courses at Macquarie University, and then at the Aengus Kavanagh Centre which is a division of the Catholic Education Office, Parramatta, a suburb of Sydney, Australia.

Initially, a group of 20 students deemed suitable for enrichment from Years 7-10 were selected by their science teachers. They were allocated $2 \times 50$ minute lessons each week for the school year. The author designed a Moodle course for the students, which contained all of the documents the students required as well as an open-ended forum to allow discussion. The program ran for four (4) years and increased the numbers of students selecting Physics for their senior studies. Many of the students then went on to pursue Science courses at tertiary institutions, which was an excellent outcome for such a program. Two images produced by students in this program are shown in Figure 1. The program came to end when the author moved to another school, St. Paul's Grammar School, Cranebrook.

\section{Astronomy and the IB}

St. Paul's Grammar School offers the International Baccalaureate from Kindergarten to Year 12. The International Baccalaureate (IB) (International Baccalaureate Organisation, 2017) aims to develop inquiring, knowledgeable and caring young people who help to create a better and more peaceful world through intercultural understanding and respect. Established in 1968, the International Baccalaureate (IB) Diploma Programme (DP) was the first programme offered by the IB and is taught to students aged 16-19. The Diploma Programme is a rigorous pre-university course. As of 16 March 


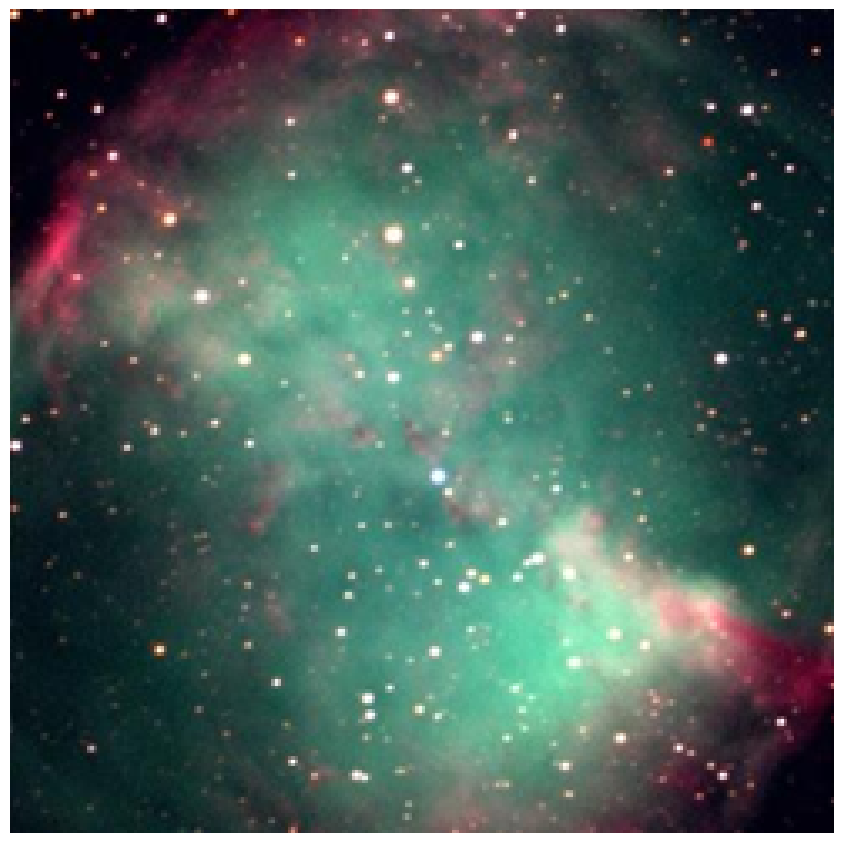

Adam M67

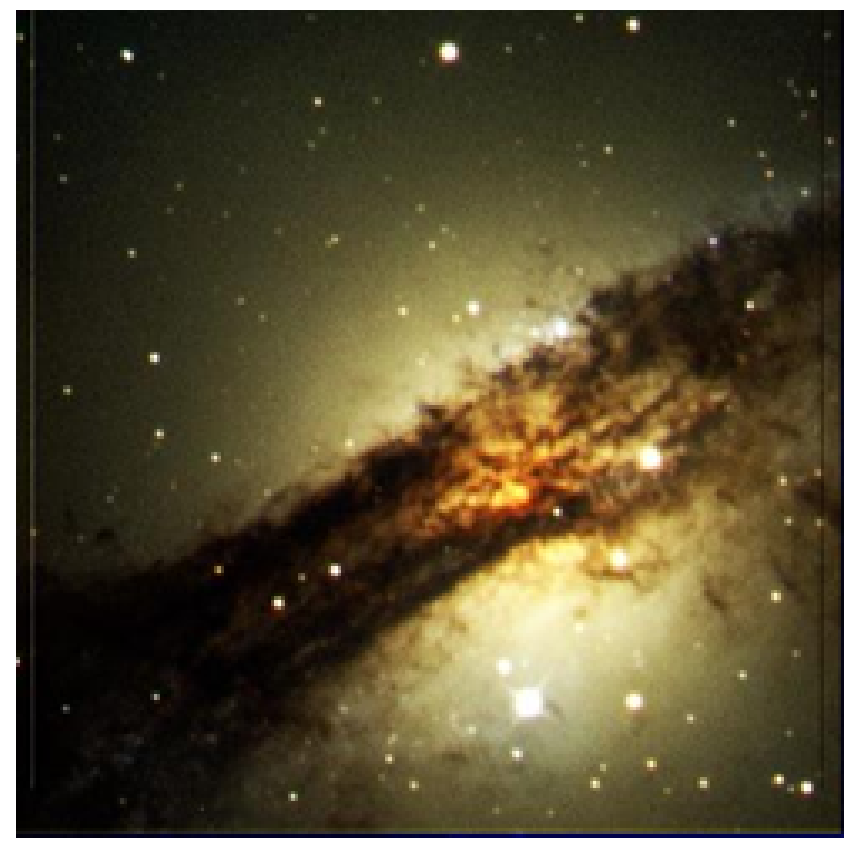

Jorge NGC5128

Figure 1. BVR Images made by two students involved in an earlier project. Both from the $2 m$ Faulkes Telescopes.

2017 , there are 3,104 schools offering the DP, in 147 different countries worldwide (International Baccalaureate Organisation, 2017)

The IB DP Physics course is a general syllabus aimed at University admission and includes traditional subjects such as Mechanics, Electricity, Magnetism, Waves, and Nuclear Physics (International Baccalaureate Organisation, 2017). The students also have to complete one option one of which is Astrophysics. The Astrophysics Option D covers stellar quantities, stellar characteristics, stellar evolution and cosmology. As such, OSS was used as the primary curriculum tool to deliver the option as it covered the syllabus completely. A comparison between the Astrophysics Option and OSS is shown below in Table 1. It's important to note that the IB Physics course is separated into Standard Level (SL) and Higher Level (HL) Physics. All students complete the SL course and selected students complete the additional HL work. Consequently, the HL exams are longer and worth more marks in the final calculations at the end of Year 12.

The Astrophysics option was delivered over 10 weeks, which is approximately 50 hours of instruction. The students were given a common research task analysing the distance and age of NGC2420. This was completed as homework and submitted as a formal practical task. In addition, many students continued with their research and submitted work for their internal assessment, which is described in the next section. An image of the class hard at work is provided in Figure 2.

\section{IB Physics - Internal Assessment - IA}

The Internal Assessment (IA), worth $20 \%$ of the final assessment in Physics, consists of one scientific investigation over a 12 month period. The individual investigation should cover a topic that is commensurate with the level of the course of study and can be a combination of the following tasks:

- a hands-on laboratory investigation

- using a spreadsheet for analysis and modelling

- extracting data from a database and analysing it graphically 
Table 1. IB Option D: Astrophysics - Components covered by OSS

\section{SL D. 1 - Stellar quantities}

Essential idea: One of the most difficult problems in astronomy is coming to terms with the vast distances between stars and galaxies and devising accurate methods for measuring them.

Understandings:

- Objects in the universe

- The nature of stars

- Astronomical distances

- Stellar parallax and its limitations

- Luminosity and apparent brightness

Applications and skills:

- Identifying objects in the universe

- Qualitatively describing the equilibrium between pressure and gravitation in stars

- Using the astronomical unit (AU), light year (ly) and parsec (pc)

- Describing the method to determine distance to stars through stellar parallax

- Solving problems involving luminosity, apparent brightness and distance

SL D. 2 - Stellar characteristics and stellar evolution

Essential idea: A simple diagram that plots the luminosity versus the surface temperature of stars reveals unusually detailed patterns that help understand the inner workings of stars. Stars follow well-defined patterns from the moment they are created out of collapsing interstellar gas, to their lives on the main sequence and to their eventual death.

Understandings:

- Stellar spectra

- Hertzsprung-Russell (HR) diagram

- Mass-luminosity relation for main sequence stars

- Stellar evolution on HR diagrams

- Red giants, white dwarfs, neutron stars and black holes

Applications and skills:

- Explaining how surface temperature may be obtained from a star's spectrum

- Explaining how the chemical composition of a star may be determined from the star's spectrum

- Sketching and interpreting HR diagrams

- Identifying the main regions of the HR diagram and describing the main properties of stars in these regions

- Applying the mass-luminosity relation

- Describing the evolution of stars off the main sequence

- Describing the role of mass in stellar evolution 
Table 1. (con't) IB Option D: Astrophysics - Components covered by OSS

HL D.4 - Stellar processes

Essential idea: The laws of nuclear physics applied to nuclear fusion processes inside stars determine the production of all elements up to iron.

Understandings:

- Nuclear fusion

- Nucleosynthesis off the main sequence

- Type Ia and II supernovae

Applications and skills:

- Describing the different types of nuclear fusion reactions taking place off the main sequence

- Applying the mass-luminosity relation to compare lifetimes on the main sequence relative to that of our Sun

- Distinguishing between type Ia and II supernovae

SL D.3 - Cosmology

Essential idea: The Hot Big Bang model is a theory that describes the origin and expansion of the universe and is supported by extensive experimental evidence.

Understandings:

- The Big Bang model

- Hubble's law

Applications and skills:

- Describing both space and time as originating with the Big Bang

- Estimating the age of the universe by assuming a constant expansion rate

HL D.5 - Further cosmology

Essential idea: The modern field of cosmology uses advanced experimental and observational techniques to collect data with an unprecedented degree of precision and as a result very surprising and detailed conclusions about the structure of the universe have been reached.

Understandings:

- The cosmological principle

- Rotation curves and the mass of galaxies

- Dark matter

- The cosmological origin of redshift

Applications and skills:

- Describing the cosmological principle and its role in models of the universe

- Describing rotation curves as evidence for dark matter

- Deriving rotational velocity from Newtonian gravitation

- Sketching and interpreting graphs showing the variation of the cosmic scale factor with time 


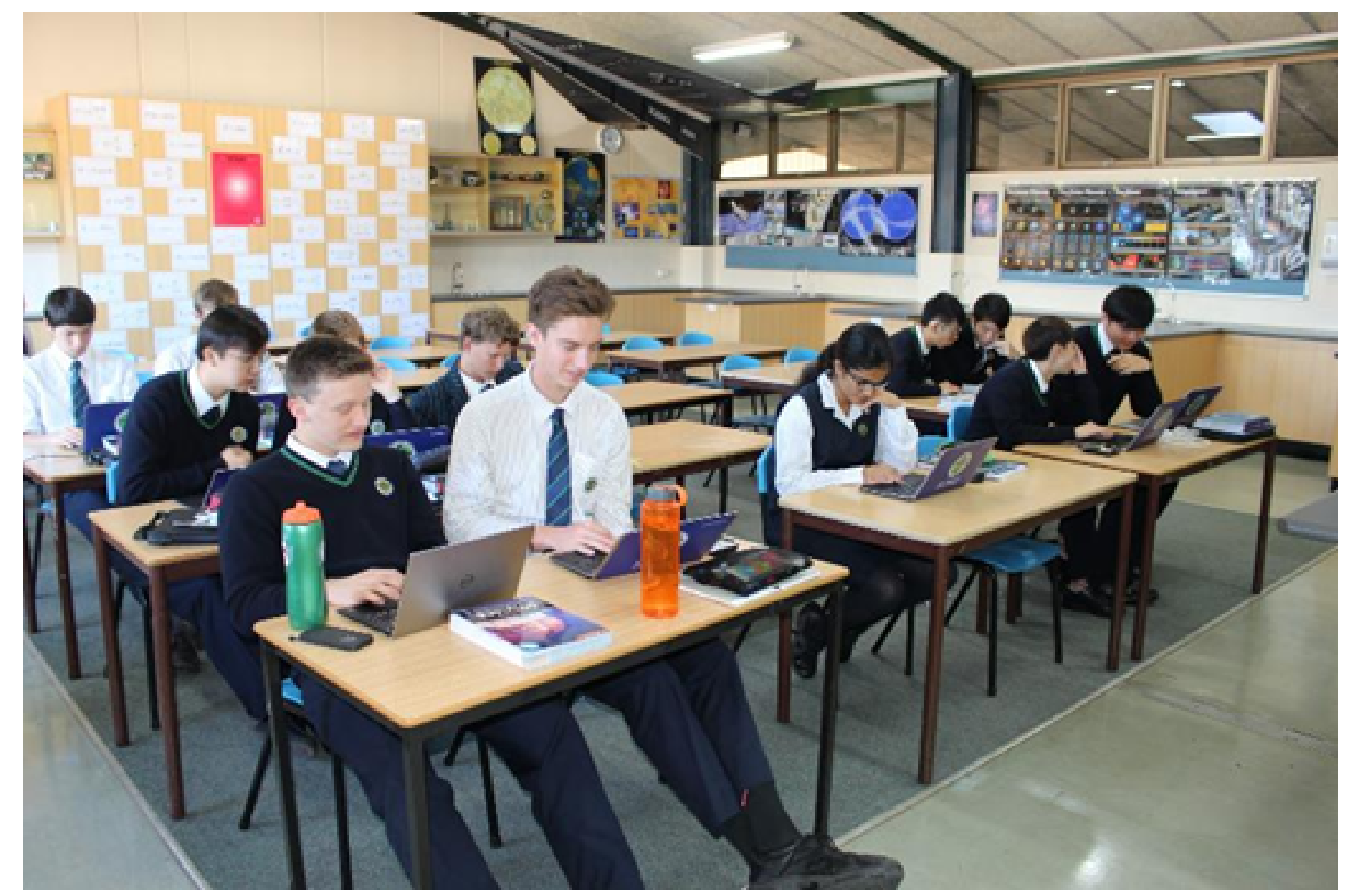

Figure 2. Year 12 IB DP Physics class hard at work

- producing a hybrid of spreadsheet/database work with a traditional hands-on investigation

- using a simulation, provided it is interactive and open-ended.

Student research, using information obtained from robotic telescopes, affords students the opportunity to use spreadsheets, databases, images, and astronomical software to research and analyse objects in the Universe. OSS was then used as the preliminary training platform to allow students to gain the skills and knowledge required to complete their internal assessments.

\section{First attempt at the IA: Open clusters (2016)}

In 2016, the first group of students completed the following projects (described in more detail in Fitzgerald et al. 2015) in OSS:
1. Telescopes, the Night Sky and the Cosmos: The students were introduced to the fundamental goal of the projects. They were then given a brief introduction to telescopes and what they do and, using a jigsaw approach, to find out the types of objects in the night sky. Students presented work on the components of the Universe, and explored sensitivity and resolution of telescopes. Many of the students then investigated planning an observation session using the Las Cumbres Observatory Global Telescope Network (Brown et al., 2013).

2. Understanding the Universe through Colour: The students looked at the nature of colour and colour imaging. The core activity was the creation of an astronomical colour image from the monochrome filtered $\mathrm{B}, \mathrm{V}$ and $\mathrm{R}$ images of their object. They also learned transferable skills in image processing (using 
GIMP or Photoshop). The students are always excited when they produce their first colour image. Some of the students also progressed to investigate the supernovae and galaxy sections of the project.

3. Uncovering the Nature and Lives of Stars: This was by far the most challenging project. The students began by investigating star clusters and their representative color magnitude diagrams (CMD). They then analysed an Open Cluster, NGC2420, where they acquired the brightness of the same stars in B, V and R filters using Aperture Photometry Tool (Laher et al. 2012a; 2012b), students learned the various methodologies and techniques used to examine various properties that can emerge from their own measured data, such as distance, age, reddening, size, proper motion, radial velocity and metallicity. These techniques were then related back to the IB Physics Astronomy option.

The students then progressed to investigating their own Open Clusters for their high level individual assessments, including NGC2302, NGC2306, NGC2354, NGC2383, NGC2384, NGC2547 and NGC 2669.

Results from two of the clusters, NGC2354 and NGC2547, can be seen below in Figures 3 and 4 respectively. The students concerned spent many hours working on their research papers. The results were very encouraging, as the results for NGC2547 were very close to the published results (Clariá, 1982). NGC2354 was further away from the published results and deserves further investigation. Overall, the students experienced real scientific research in collaboration with university academics, particularly the OSS staff shown in Figure 5, who visited the school on a number of occasions to impart knowledge and skills the students required. An image of the students' work is shown in Figure 6.

\section{Extended Essay}

In the Diploma Programme, the Extended Essay $(E E)$ is the prime example of a piece of work where the student has the opportunity to show knowledge, understanding and enthusiasm about a topic of his or her choice. The extended essay is an in-depth study of a focused topic presented in a 4,000 word report and is intended to promote high-level research and writing skills, intellectual discovery and creativity. It provides students with an opportunity to engage in personal research, under the guidance of a supervisor. This leads to a major formal report presented with structured writing in which ideas and findings are communicated in a reasoned and coherent manner, appropriate to the subject chosen.

\section{The aims of the extended essay are to provide students with the opportunity to:}

- pursue independent research on a focused topic

- develop research and communication skills

- develop the skills of creative and critical thinking

- engage in a systematic process of research appropriate to the subject

- experience the excitement of intellectual discovery (International Baccalaureate Organisation, 2017)

\section{In working on the extended essay, students are} expected to:

- plan and pursue a research project with intellectual initiative and insight

- formulate a precise research question

- gather and interpret material from sources appropriate to the research question

- structure a reasoned argument in response to the research question on the basis of the material gathered 


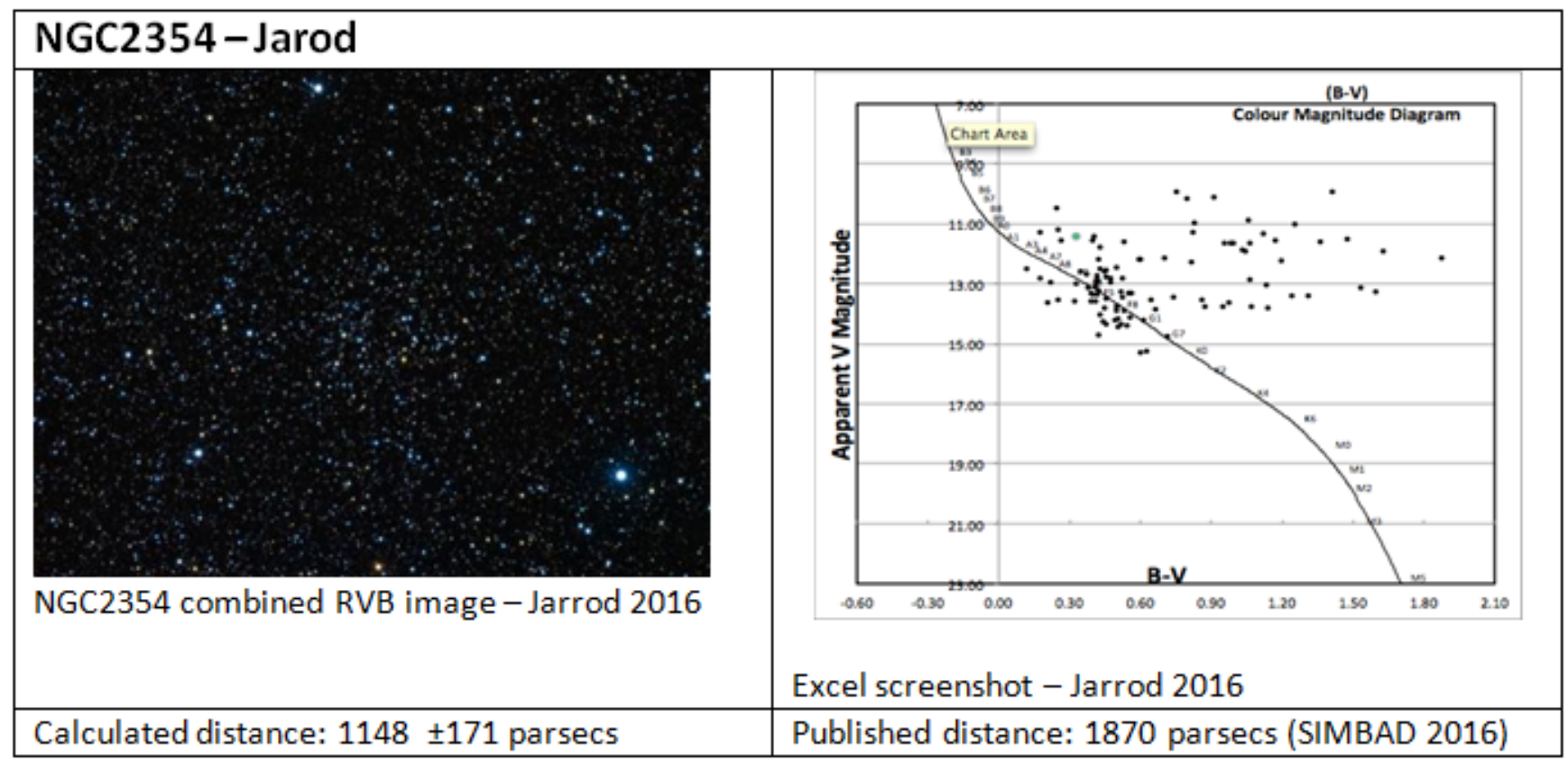

Figure 3. Jarod's image and CMD of NGC 2354 from his IA IB project

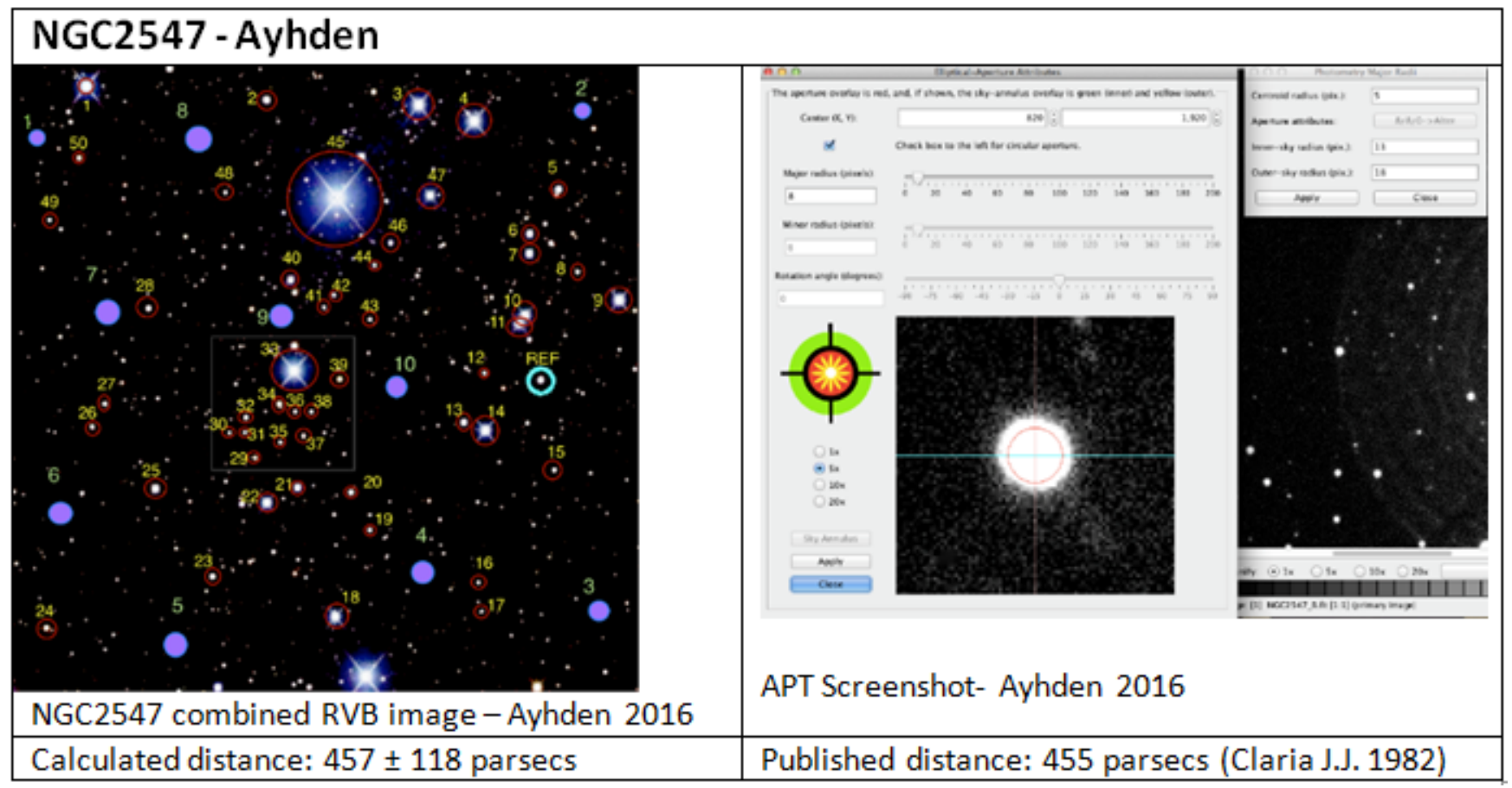

Figure 4. Ayhden's image and a screenshot of APT during the measurement process from his IA IB 


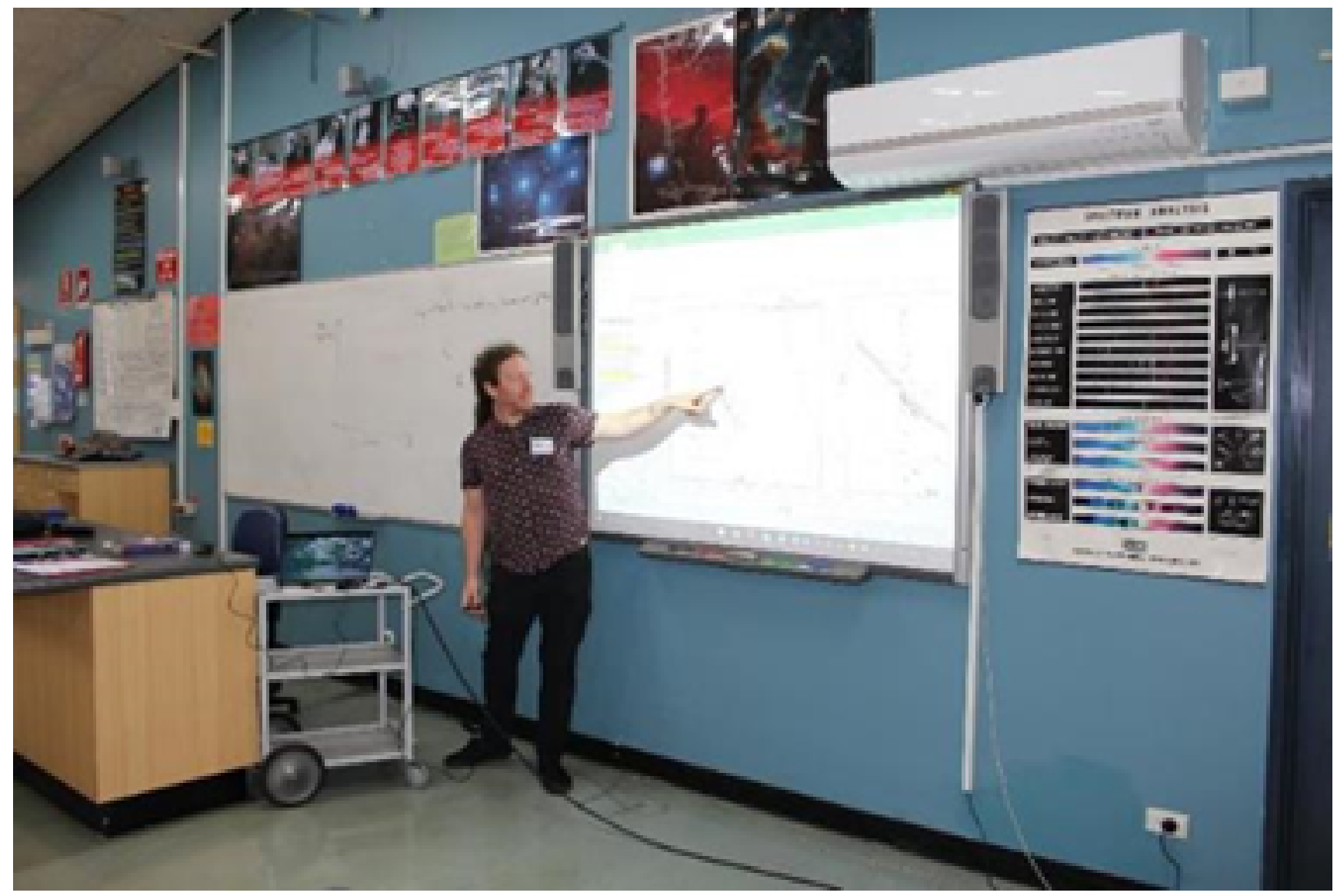

Figure 5. Dr. Fitzgerald, the OSS mentor, explaining Colour Magnitude Diagrams
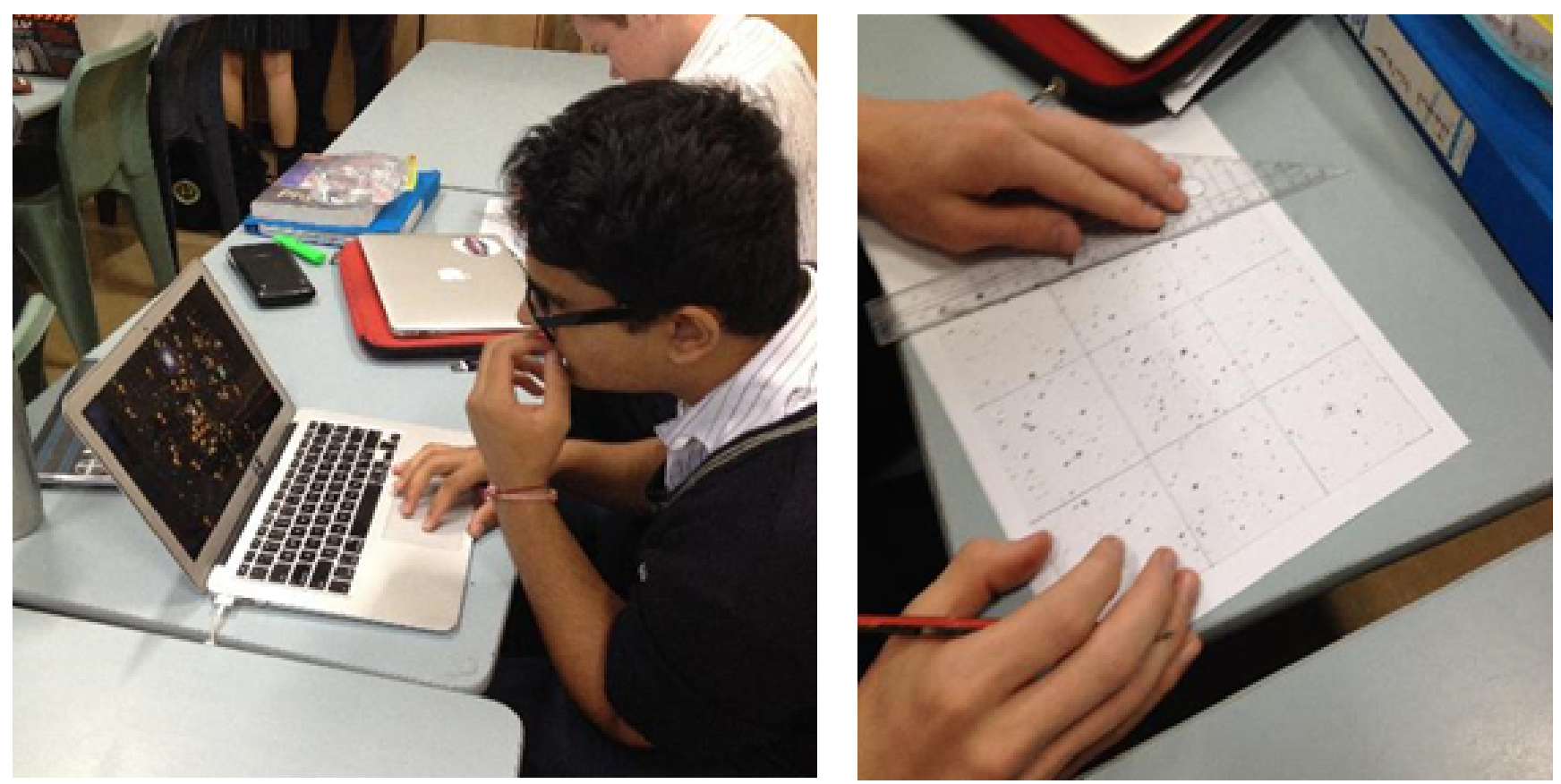

Figure 6. Ayhden and Jarod analyzing their Open Clusters 
- present their extended essay in a format appropriate to the subject, acknowledging sources in one of the established academic ways

- use the terminology and language appropriate to the subject with skill and understanding

- apply analytical and evaluative skills appropriate to the subject, with an understanding of the implications and the context of their research (International Baccalaureate Organisation, 2017)

\section{Extended essays researching open clusters in 2016}

In 2016, two students completed their extended essays on:

- the determination of the distance to Open Cluster NGC2383

Lachlan calculated the distance to NGC2383 to be 1752pc using the software and techniques he learnt in OSS. His results were close to the published value of $1655 \mathrm{pc}^{1}$. Considering the distance was calculated nearly 20 years ago, it might be considered that Lachlan's value may be closer to the actual value.

\section{- the determination of the distance of Open} Cluster NGC2384

Yusen used the software and techniques he learned in OSS and calculated the distance to NGC2384, as shown in Figure 7, to be 2754 $\mathrm{pc}$, which is farther than the published value of $2116 \mathrm{pc}^{2}$. Considering the distance was calculated over 50 years ago, it might be considered that Yusen's value may be closer to the actual value and is worthy of further investigation.

\footnotetext{
${ }^{1}$ https://www.univie.ac.at/webda/cgi-bin/ocl_page.cgi? dirname $=$ ngc 2383

${ }^{2}$ https://www.univie.ac.at/webda/cgi-bin/ocl_page.cgi? dirname $=$ ngc 2384
}

The students were extremely motivated and completed their research on time. They were both awarded good marks by the IB Organisation and went on to pursue Science degrees at University.

\section{Extended essays researching broader topics than open clusters in 2017}

In late 2016, a wider range of topics was suggested by the OSS mentor. The scope of the research would go beyond the material covered in OSS and the IB, and be of university research standards. Primarily the students would be fulfilling their IB requirements, and also be contributing to real-world research.

The students worked collaboratively with the authors. Each student was assigned a Moodle course, which allowed them to access all the academic articles and resources they required. It also enabled each student to have their own forum to discuss issues. In addition, the OSS mentor produced instructional videos to illustrate the harder concepts, including specialised astronomy software such as Aperture Photometry Tool (Laher et al. 2012a, 2012b), Peranso (Paunzen and Vanmunster, 2013) and PysoChrone (Fitzgerald, 2018).

Four students began their extended essays at the end of 2016. The OSS mentor visited the students a number of times, and held online meetings to assist the students with their research. Their topics included:

\section{- Whether Riddle 15 is an Open or Globular Cluster (Andrew)}

Andrew concluded that Riddle 15, the stellar group shown in Figure 8, is an Open Cluster. He analysed the shape, location and stellar types to back up his conclusion. Presently, there is no research data for this cluster, so his results are important for further research.

\section{- re-observing Hubble's First Variable Star in the modern era (Michael)}

Michael analysed the first Cepheid variable star, M31-V1, observed by Edwin Hubble in 1925. Very 

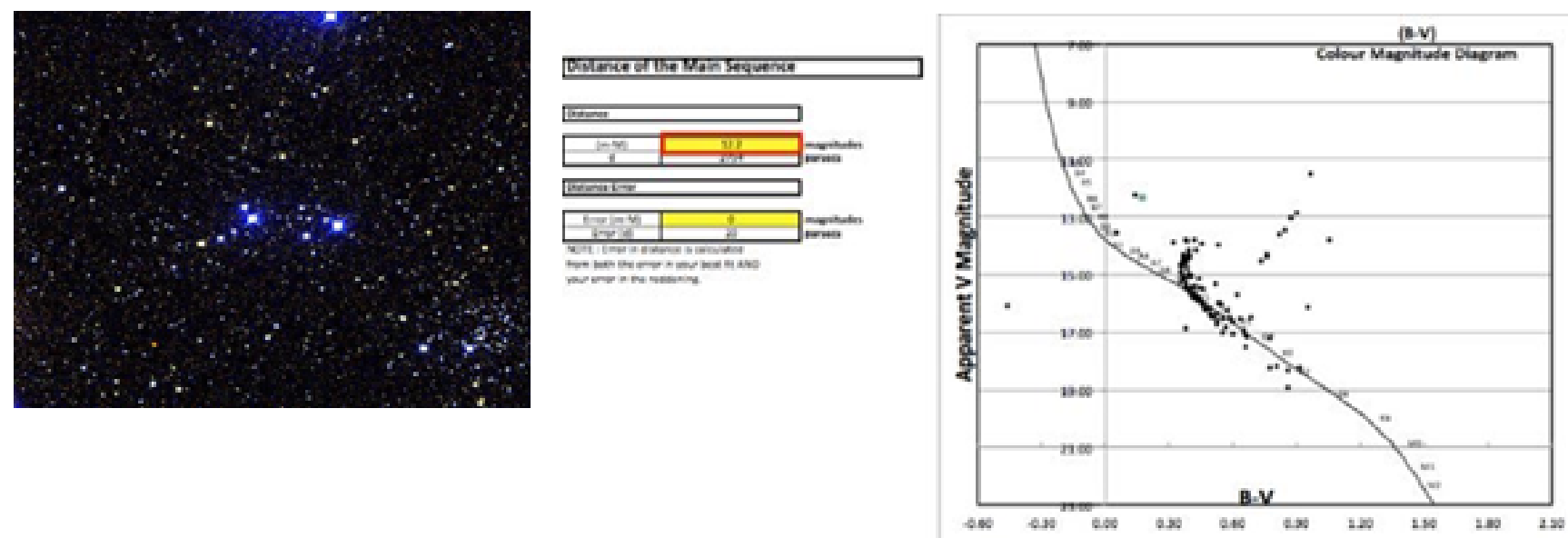

Figure 7. Yusen's colour image and CMD of Open cluster 2384.

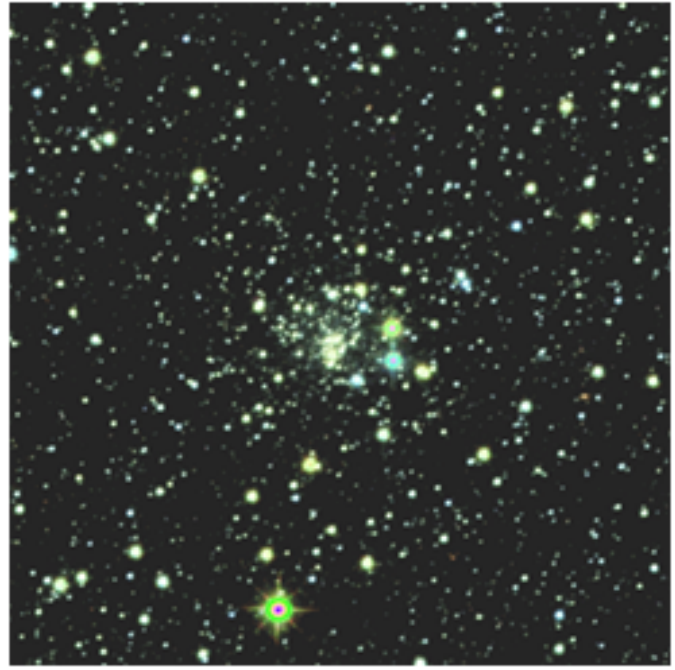

Figure 8. Andrew's Riddle 15 colour image processed using Fits Liberator and Adobe Photoshop from $R$, $V$, B images (LCOGTn)

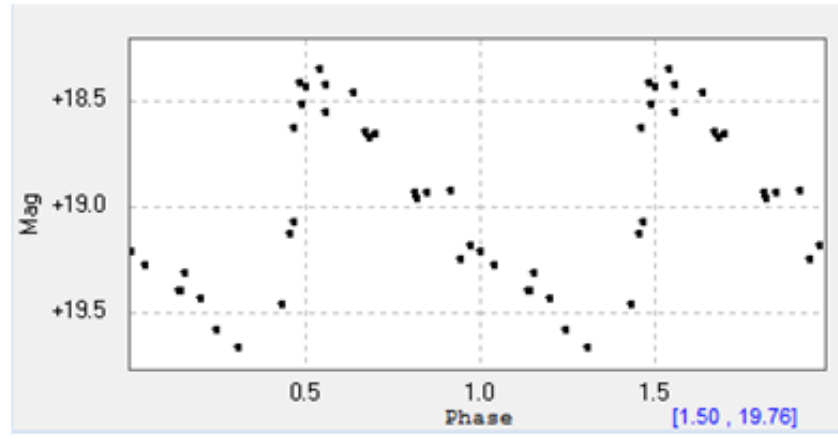

Figure 9. Michael's B-band Peranso light curve shown with a period of $(31.4301 \pm 0.03182)$ little research has been done since then, so he was determined to see if Hubble's original results still held up to academic scrutiny.

Michael produced results that showed the period to be $31.4301 \pm 0.03182$ days with the $\mathrm{B}$ band light curve shown in Figure 9. This closely agreed with the results calculated by Edwin Hubble of 31.41 days, and lies within the uncertainty of later research in the 1950s by Baade and Swopes of 31.384 days. By using B, V and I filters, he was able to independently estimate the reddening towards the star and the distance $807 \mathrm{kpc}$ which was very close to the accepted modern value of 766kpc (Templeton et al., 2011).

Michael's results confirmed Hubble's original observations and will be used in further study of M31.

\section{- identifying candidate Binary Open clusters for future study (Toby)}

Toby analysed over 200 possible open clusters to determine if they were gravitationally bound to one another, and therefore true binary open clusters. To do this, he compared values of distance, age, $\mathrm{E}(\mathrm{B}-\mathrm{V})$, angle separation and total separation using a pair-matching code and the Dias et al. (2002) open cluster catalogue. The ten most likely binary open cluster candidates were identified for future study and are shown in Table 2. 

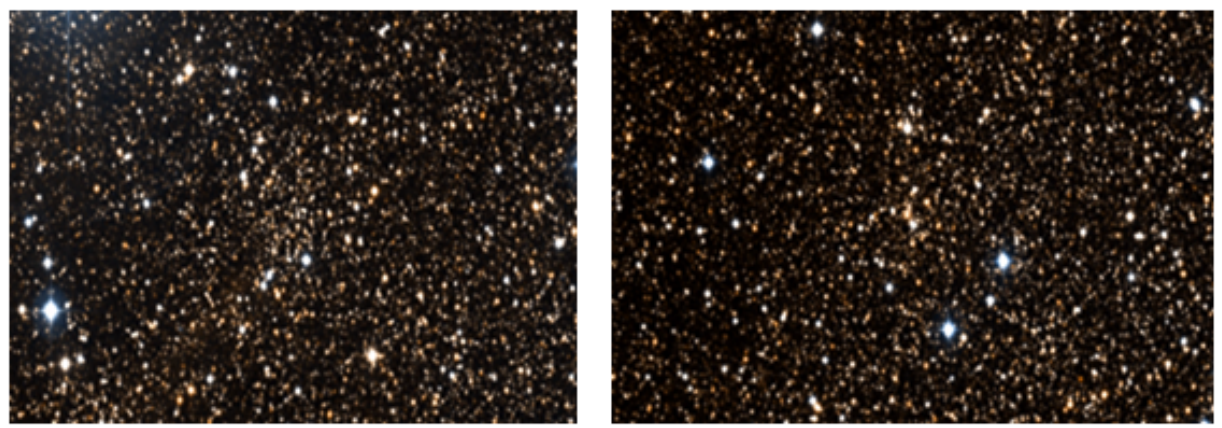

Figure 10. A pair of potential binary open clusters from Toby's IB Extended Essay

Table 2. Pairs of open clusters identified in Toby's IB Extended Essay study.

\begin{tabular}{|c|c|}
\hline Kronberger 52 and Kronberger 68 & Alessi 8 and Johansson 1 \\
\hline NGC 3324 and NGC 3294 & NGC 6169 and NGC 6178 \\
\hline NGC 6840 and NGC 6843 & Ruprecht 50 and Ruprecht 153 \\
\hline Sigma Orionis and Trapezium & FSR 0977 and FSR 0979 \\
\hline Hogg 20 and Hogg 21 & Ruprecht 50 and Ruprecht 153 \\
\hline
\end{tabular}

As example of his work for Kronberger 52 and Kronberger 68 is shown in Table 3 below. An image of these clusters is shown in Figure 10. He repeated the analysis for over 200 pairs of Open Clusters and reduced the list to the above ten pairs. Further research will now be done by professional astronomers in the light of his findings.

\section{- Variables in NGC1261 (Scott)}

Scott identified 23 RR Lyrae variable stars in the globular cluster, NGC1261 (shown in Figure 11) with images taken from the 1-meter telescope at the Siding Springs Observatory in New South Wales, Australia, at irregular intervals over a time period of 61 days. He then calculated the distance to the cluster using software and techniques learnt in OSS, a sample periodogram and lightcurve is shown in Figure 12. By focusing on the RR Lyraes, he was able to estimate the distance of $16.444 \pm 1.213 \mathrm{kpc}$ using the i-band and z-band Period-Luminosity-Metallicity relationship for RR Lyraes (Cáceres and Catelan, 2008). This result is in line with recent distance calculations of $16.4 \mathrm{kpc}$ (Paust et al., 2010).

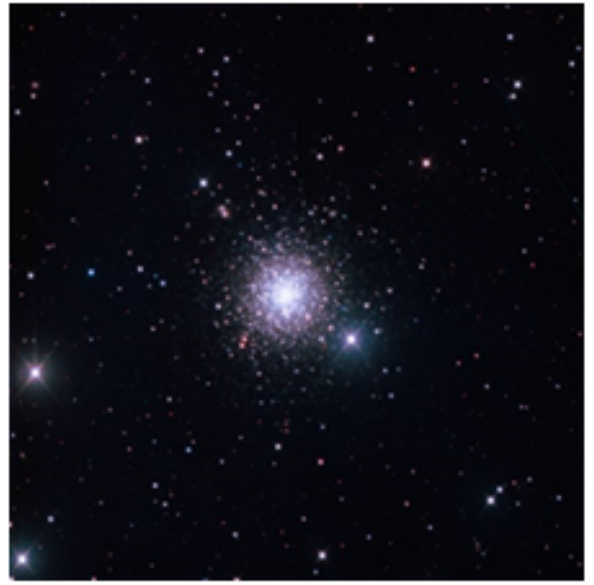

Figure 11. Scott's colour image of NGC1261 colour image processed using Fits Liberator and Adobe Photoshop from R, V, B images (LCOGTn)

Since this was the first use of real data with the given relationships, the techniques employed by Scott were indeed ground breaking. The data and research from his investigation will now be used to determine more accurate knowledge of the characteristics of RR Lyrae variable stars, and develop more precise analyses of them to help understand the structure of the Milky Way Galaxy. Scott has also agreed to work on this research after 
Table 3. An example of the results Toby found using Pysochrone for a sample pair of clusters

\begin{tabular}{|c|c|c|c|c|}
\hline Name & Age/ years & E(B-V) & Dist/ parsecs & $(\mathrm{m}-\mathrm{M})$ \\
\hline Kronberger 52 & $1.25 \cdot 10^{8} \pm 1.7 \cdot 10^{7}$ & $1.77 \pm 0.01$ & $794 \pm 0.44$ & $9.5 \pm 0.01$ \\
\hline Kronberger 68 & $1.25 \cdot 10^{8} \pm 1.7 \cdot 10^{7}$ & $1.7 \pm 0.01$ & $794 \pm 0.76$ & $9.5 \pm 0.01$ \\
\hline
\end{tabular}
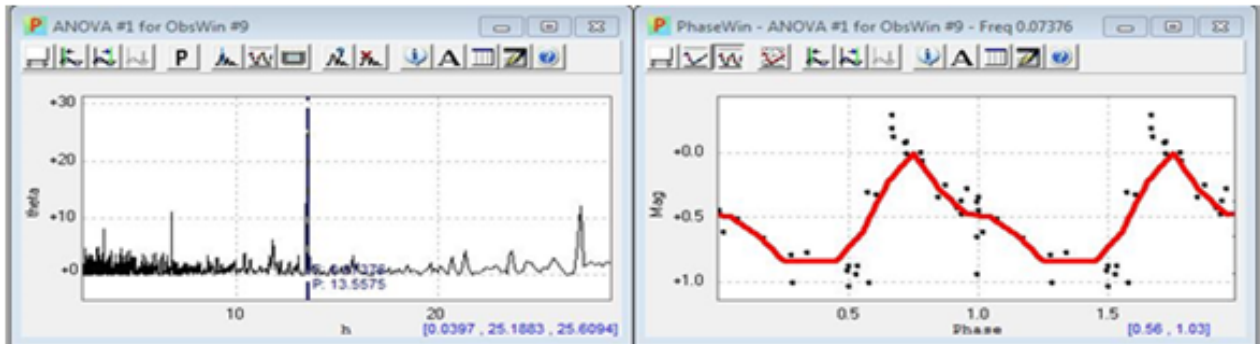

Figure 12. Typical representation of one of the star's period determination and apparent magnitude/period graph using the Peranso software

he finishes his final IB examinations for publication.

\section{Discussion}

The author has been teaching the IB Physics course for the past 3 years, and attended professional development courses aimed at delivering the Diploma Programme with IB teachers from around the world. In all discussions and internet searches, there is no evidence that IB Physics teachers are using astronomy research as part of the IB Physics course, either as an internal assessment or an extended essay. Therefore, astronomy research had to be implemented from previous experience, mainly with the OSS project over the past eight (8) years.

Initially OSS was delivered as an alternative to the traditional chalk and talk IB Astrophysics Option. This was completed in 8 weeks of normal instruction during Term 4 each year. The match between the IB syllabus and OSS material is very close, as shown in the section IB Option D: Astrophysics - Components covered by OSS. Consequently, there was very little extra instruction required by the author, other than completing past examination questions as revision exercises.

The students were then given a number of research topics that would suit either their IA or EE. Some of these topics were provided by the OSS mentor. Once these were discussed at length with the author, the students began their research over the summer holidays. Moodle courses were set up for the students, with the author and the OSS mentor as the teachers. The students used the forums in Moodle to ask questions over the summer break. The students went on to complete their IAs or EEs over the next six months.

The main difficulties experienced were time and knowledge. Time is at a premium in schools, and there are many competing demands on students. Many of the students were able to complete their research during vacation times with the help of the author and the OSS mentor. Knowledge was also a stumbling block. The OSS mentor was able to engage in remote video sessions to assist the students on the finer points of their research. He also produced a number of instructional videos as the need arose. The author was also required to learn a large amount of astrophysics knowledge about the research topics and the specialised software used. The author had engaged in, and delivered OSS training to teachers in the past, so this was not an onerous task, but it would likely be a stumbling block for the average Physics teacher.

On the positive side, there were many benefits from the research. Firstly, student engagement 
dramatically increased. The delivery of the OSS material saw the students actively questioning the author about astronomy concepts beyond the IB course. Over half of the students then went on to do astronomy research for their IA or EE, which was very encouraging. Secondly, many of the parents expressed their delight at the amount of interest displayed by their children at home. Finally, the school was very supportive, with the principal in particular visiting the IA and EE classes and commenting on the advanced research the students were taking on.

In hindsight, there have been a few lessons learned from the process. The main issue is that the teacher needs to be confident in delivering OSS and then supervising the IA or EE. Consequently, teacher training is mandatory, as few Physics teachers possess the astronomy research knowledge and skills needed to implement such a program. Over the past few years the OSS staff have delivered training to teachers across Australia. This has seen an increase in the number of students attempting astronomy research and a keen band of Physics teachers equipped with the skills required to do further research.

In addition, the process could not have been completed without an academic mentor assisting the author and students. The level of material was beyond the IB course and required specialised university astronomy knowledge and skills. Constant support from the mentor using the Moodle forums, meant that the students were able to understand and to interpret their results, leading to the possibility of some of the student's research being cited in future academic papers. The role of the mentor enabled the students to complete their research in a professional and timely manner. The mentor was also seen as a guide for students pursuing astronomy as an academic pursuit.

Timing of the delivery of OSS, and then the IA or EE, has proven to be critical. The skills required for further research needed to be consolidated and refined before the students could attempt their research projects. After a number of years, it has been narrowed down to delivering OSS at the end of the first senior year, Year 11, with research being carried into the summer break. This gives the students the time required and sets up a good platform for completing the IA or EE early in their second and final year of the IB.

As discussed, the IB Physics course lists Astrophysics as an option for the two year course. Interestingly, astronomy concepts are covered to varying degrees throughout the compulsory core components. Table 4 presents a general overview of the components, both Standard and Higher Level, where astronomy is integral to the information being delivered in the unit. As can be seen, astronomical concepts are spread over the entire course, and in many instances, garner improved student interest and interaction. The author has used OSS projects: 1-Telescopes, the Night Sky and the Cosmos and 2-Understanding the Universe through Colour, to enhance and extend students in Topics 4, 6, 7, 9, and 12. The students have responded positively to the inquiry-based approach and particularly enjoyed producing images of stellar objects from remote telescopes.

Breaking new ground is never easy, but the IB Physics course is designed to be thought provoking and inquiry-based. Although there have been some difficulties, the positive influence on student engagement and learning has been highly beneficial. With the necessary training, IB Physics teachers would find this approach both achievable and educationally sound.

\section{Conclusions}

Astronomy research has a real and justifiable place in the International Baccalaureate Diploma Physics course. The inquiry-based approaches using robotic telescopes are a perfect match for the creative and critical thinking skills required in the IB. Over the years, the students have gained skills and knowledge that has taken them to tertiary science courses around the world. It has taken energy and enthusiasm to pursue such an approach, but the end result has been to witness a group of students who have achieved outcomes far beyond 
Table 4. Astronomy Concepts in the IB Physics Course - Core Components

\begin{tabular}{|c|c|c|c|}
\hline $\begin{array}{l}\text { Standard Level } \\
\text { (SL) }\end{array}$ & & $\begin{array}{c}\text { Additional higher level } \\
\text { (AHL) }\end{array}$ & \\
\hline \multirow[t]{4}{*}{ Topic 2: Mechanics } & $2.1-$ Motion & & \\
\hline & $2.2-$ Forces & & \\
\hline & 2.3 - Work, energy and power & & \\
\hline & 2.4-Momentum and impulse & & \\
\hline \multirow[t]{4}{*}{ Topic 4: Waves } & 4.3 - Wave characteristics & $\begin{array}{l}\text { Topic 9: Wave } \\
\text { phenomena }\end{array}$ & $\begin{array}{l}9.2 \text { - Single-slit } \\
\text { diffraction }\end{array}$ \\
\hline & $4.4-$ Wave behaviour & & $9.3-$ Interference \\
\hline & & & 9.4 - Resolution \\
\hline & & & 9.5 - Doppler effect \\
\hline \multirow[t]{2}{*}{$\begin{array}{c}\text { Topic 6: Circular motion } \\
\text { and gravitation } \\
\end{array}$} & 6.1 - Circular motion & & \\
\hline & $\begin{array}{c}6.2-\begin{array}{l}\text { Newton's law of } \\
\text { gravitation }\end{array}\end{array}$ & & \\
\hline \multirow[t]{2}{*}{$\begin{array}{c}\text { Topic 7: Atomic, } \\
\text { nuclear and }\end{array}$} & 7.2 - Nuclear reactions & $\begin{array}{l}\text { Topic 12: Quantum } \\
\text { and nuclear physics }\end{array}$ & $\begin{array}{l}\text { 12.1 - The interaction of } \\
\text { matter with radiation }\end{array}$ \\
\hline & $\begin{array}{c}7.3 \text { - The structure of } \\
\text { matter }\end{array}$ & & 12.2 - Nuclear physics \\
\hline \multirow[t]{2}{*}{$\begin{array}{l}\text { Topic 8: Energy } \\
\text { production }\end{array}$} & 8.1 - Energy sources & & \\
\hline & 8.2 - Thermal energy & & \\
\hline
\end{tabular}

the normal expectations of senior high school Physics students.

\section{Acknowledgements}

The majority of the astronomy research conducted by the author has been achieved using Our Solar Siblings, designed by Dr. Michael Fitzgerald, Prof. David McKinnon and Dr. Lena Danaia. Thanks must go to their dedication since 2009 to design and refine the course materials, and to train teachers across Australia.

Dr. Michael Fitzgerald must also be thanked for the huge amount of work he has put into assisting the students with their internal assessments and extended essays. The results are worthy of further research, and hopefully publication in the future.

St. Paul's Grammar School also needs to be thanked for allowing the author to deliver astronomy research and welcoming visiting academics to the school.

\section{References}

Bailey, J. M. (2011). Astronomy education research: Developmental history of the field and summary of the literature. Commissioned paper for the National Research Council Board on Science Education's Committee on the Status, Contributions, and Future Directions of Discipline Based Education Research.

Bailey, J. M. and Lombardi, D. (2015). Blazing the trail for astronomy education research. Journal of Astronomy and Earth Sciences Education, 2(2):77.

Brown, T., Baliber, N., Bianco, F., Bowman, M., Burleson, B., Conway, P., Crellin, M., Depagne, É., De Vera, J., Dilday, B., et al. (2013). Las Cumbres Observatory global telescope network. Publications of the Astronomical Society of the Pacific, 125(931):1031. 
Cáceres, C. and Catelan, M. (2008). The periodluminosity relation of RR Lyrae stars in the SDSS photometric system. The Astrophysical Journal Supplement Series, 179(1):242.

Clariá, J. (1982). Membership Basic Parameters and Luminosity Function of the Southern Open Cluster NGC2547. Astronomy and Astrophysics Supplement Series, 47:323.

Danaia, L., Fitzgerald, M., and McKinnon, D. (2013). Students' perceptions of high school science: what has changed over the last decade? Research in Science Education, 43(4):1501-1515.

Danaia, L., McKinnon, D., Parker, Q., Fitzgerald, M., and Stenning, P. (2012). Space to grow: LCOGT.net and improving science engagement in schools. Astronomy Education Review, 11(1).

Dias, W., Alessi, B., Moitinho, A., and Lépine, J. (2002). New catalogue of optically visible open clusters and candidates. Astronomy \& Astrophysics, 389(3):871-873.

Fitzgerald, M. (2018). The Our Solar Siblings Pipeline: Tackling the data issues of the scaling problem for robotic telescope based astronomy education projects. In Fitzgerald, M. T., James, C. R., Buxner, S., and White, S., editors, Robotic Telescopes, Student Research, and Education Proceedings, volume 1(1), pages 343354.

Fitzgerald, M., Danaia, L., and McKinnon, D. H. (2017). Barriers inhibiting inquiry-based science teaching and potential solutions: perceptions of positively inclined early adopters. Research in Science Education, pages 1-24.

Fitzgerald, M., McKinnon, D. H., and Danaia, L. (2015). Inquiry-based educational design for large-scale high school astronomy projects using real telescopes. Journal of Science Education and Technology, 24(6):747-760.

Fitzgerald, M., McKinnon, D. H., Danaia, L., and Deehan, J. (2016). A large-scale inquiry-based astronomy intervention project: impact on students' content knowledge performance and views of their high school science classroom. Research in Science Education, 46(6):901-916.

Fitzgerald, M. T., Hollow, R., Rebull, L. M., Danaia, L., and McKinnon, D. H. (2014). A review of high school level astronomy student research projects over the last two decades. Publications of the Astronomical Society of Australia, 31.

Fitzgerald, M. T., McKinnon, D. H., Danaia, L., Cutts, R., Salimpour, S., and Sacchi, M. (2018). Our Solar Siblings: A high school focused robotic telescope-based astronomy education project. In Fitzgerald, M. T., James, C. R., Buxner, S., and White, S., editors, Robotic Telescopes, Student Research, and Education Proceedings, volume 1(1), pages 217-231.

Gomez, E. L. and Fitzgerald, M. T. (2017). Robotic telescopes in education. Astronomical Review, 13(1):28-68.

International Baccalaureate Organisation (2017). The International Baccalaureate: International education and cultural preservation.

Laher, R. R., Gorjian, V., Rebull, L. M., Masci, F. J., Fowler, J. W., Helou, G., Kulkarni, S. R., and Law, N. M. (2012a). Aperture photometry tool. Publications of the Astronomical Society of the Pacific, 124(917):737.

Laher, R. R., Rebull, L. M., Gorjian, V., Masci, F. J., Fowler, J. W., Grillmair, C., Surace, J., Mattingly, S., Jackson, E., Hacopeans, E., et al. (2012b). Aperture photometry tool versus SExtractor for noncrowded fields. Publications of the Astronomical Society of the Pacific, 124(917):764.

Paunzen, E. and Vanmunster, T. (2013). Peranso (Light Curve and Period Analysis Software).

Paust, N. E., Reid, I. N., Piotto, G., Aparicio, A., Anderson, J., Sarajedini, A., Bedin, L. R., Chaboyer, B., Dotter, A., Hempel, M., et al. (2010). The ACS Survey of Galactic Globular 
Clusters. VIII. Effects of Environment on Globular Cluster Global Mass Functions. The Astronomical Journal, 139(2):476.

Templeton, M., Henden, A., Goff, W., Smith, S., Sabo, R., Walker, G., Buchheim, R., Belcheva, G., Crawford, T., Cook, M., et al. (2011). Modern observations of Hubble's first-discovered Cepheid in M31. Publications of the Astronomical Society of the Pacific, 123(910):1374. 